\title{
THE CRITICAL MASS FOR THE OVERSTABILITY OF MASSIVE ZERO-AGE MAIN SEQUENCE STARS
}

\author{
J. Klapp \\ Department of Physics, Universidad Autónoma Metropolitana, \\ Iztapalapa, México 09340 DF \\ and \\ Instituto Nacional de Investigaciones Nucleares, Salazar, \\ Edo. de México, México. \\ N. Langer and K.J. Fricke \\ Universitäts-Sternwarte Göttingen, Geismarlandstraße 11, \\ D-3400 Göttingen, Federal Republic of Germany.
}

ABSTRACT. The linear stability of massive extreme Pop I stars at the main sequence is investigated with a full nonadiabatic treatment. Contrary to earlier beliefs these stars become vibrationally unstable only beyond a critical mass as high as $440 M_{\odot}$ at the main sequence.

It has been maintained now for a very long time since the classical work by Ledoux (1941) and by Schwarzschild and Härm (1959) that stars in excess of $\sim 100 M_{\odot}$ cannot exist on the ground of their overstability alone. According to these results the nuclear energizing of pulsations in the stellar core - the $\epsilon$-mechanism -- overcomes the damping and is believed to essentially disrupt the star or to cause strong mass loss which reduces their lifetimes effectively. On the other hand, evidence for the existence of stars with masses well in excess of $100 M_{\odot}$ has come from observations of the brightest stars in the galaxies of the Local Group (Humphreys, 1982; De Jager, 1980; Massey and Hutchings, 1983) and from the interpretation of Wolf-Rayet stars (Maeder, 1982), P Cygni stars and HubbleSandage variables, etc. (Humphreys, 1984). The extreme phenomena like $\eta$ Carina in our galaxy and R136a in the LMC call for main sequence masses in excess of hundred solar masses.

Motivated by the apparent discrepancy between theory and observations concerning the upper mass limit, we reconsider in this paper the linear theory for radial pulsations of massive stars. The linear stability analysis is performed with a modified version of the Los Alamos Nonadiabatic Code (LNA). The major modifications introduced to the LNA code are (i) construction of the equilibrium model and (ii) inclusion of a nuclear energy generation term into the energy equation. For our computations the equilibrium model is constructed with the Göttingen stellar evolution code (cf. Langer et al., 1985). We have taken special care in using identical input physics both in stellar evolution code and in the pulsation code.

In the LNA code a first guess to the eigenfrequency is obtained by the use of the quasiadiabatic approximation and a first order expansion about the adiabatic mode. From 
Castor (1971) it is

$$
\omega^{2}-\omega_{0}^{2}=\frac{\int_{0}^{M_{*}}\left(\delta r^{T} G_{2}\left(i \omega_{0} I-K_{2}\right)^{-1} K_{1} \delta r\right) d m}{\int_{0}^{M_{*}}(\delta r)^{2} d m},
$$

where $\omega_{0}$ and $\omega$ are the adiabatic and quasiadiabatic eigenfrequencies, respectively, $\delta r$ is the radial adiabatic eigenvector, $T$ denotes the transpose operation, $I$ is the identity matrix, $M_{*}$ is the total mass of the star, and the matrices $K_{1}, K_{2}$ and $G_{2}$ are defined by equations (13) and (14) of Castor (1971). We will call this approximation the Castor quasiadiabatic (CQAD) approximation. The LNA code then computes the nonadiabatic eigenvalues $\omega$ and the right and left radial and entropy eigenvectors satisfying the usual orthogonality condition with a precision of about one part in $10^{12}$.

The imaginary part of the eigenfrequency in Ledoux's (1941) quasiadiabatic approximation (LQAD) is calculated using the adiabatic eigenvectors by the well known expression

$$
I m(\omega)=\frac{-\frac{1}{2} \int_{0}^{M_{*}^{*}} \frac{\delta T}{T}\left(\delta \epsilon-\frac{d \delta L}{d m}\right) d m}{\omega_{0}^{2} \int_{0}^{M *}(\delta r)^{2} d m}
$$

where all quantities have their usual meanings. It is this approximation that has mostly been used to study the linear stability of massive objects (Ledoux, 1941; Schwarzschild and Härm, 1959; Stothers and Simon; 1970; Papaloizou, 1973; Noels and Masereel, 1982; and Maeder, 1985; see, however, Ziebarth, 1970).

For the stability analysis we constructed a sequence of models from $130 M_{\odot}$ to $5000 M_{\odot}$ with a chemical composition of $(X, Z)=(0.687,0.043)$.

Fig. 1 shows the imaginary part of the frequency $\omega$ against total mass for the fundamental mode as calculated using the nonadiabatic formalism (crosses) and the CQAD approximation (circles). Unstable modes correspond to negative values of $\operatorname{Im}(\omega)$. From the Figure we observe that we have reproduced Ledoux's classical result in the sense that an instability of the fundamental mode occurs for high enough masses. The major difference to the general picture is that we have encountered the instability at about $440 M_{\odot}$ rather than already at about $100 M_{\odot}$. The delay in the occurrence of the instability is due to nonadiabatic effects in the outer layers of the star. It is also interesting to note that the CQAD approximation gives an extremely good approximation to the nonadiabatic eigenfrequency specially in the low mass region, always predicting values slightly below the nonadiabatic ones.

In order to estimate the influence of the outermost nonadiabatic layers we have plotted in Fig. 2 the imaginary part of the frequency of the fundamental mode from the CQAD approximation (solid line) and the LQAD approximation (dashed line) as a function of the radius up to which the integral in equations (1) and (2) is performed.

For the LQAD approximation we observe a slow damping effect of the outer layers until the approximation gives unreasonable values. This occurs at a fractional radius of about 0.98 . 


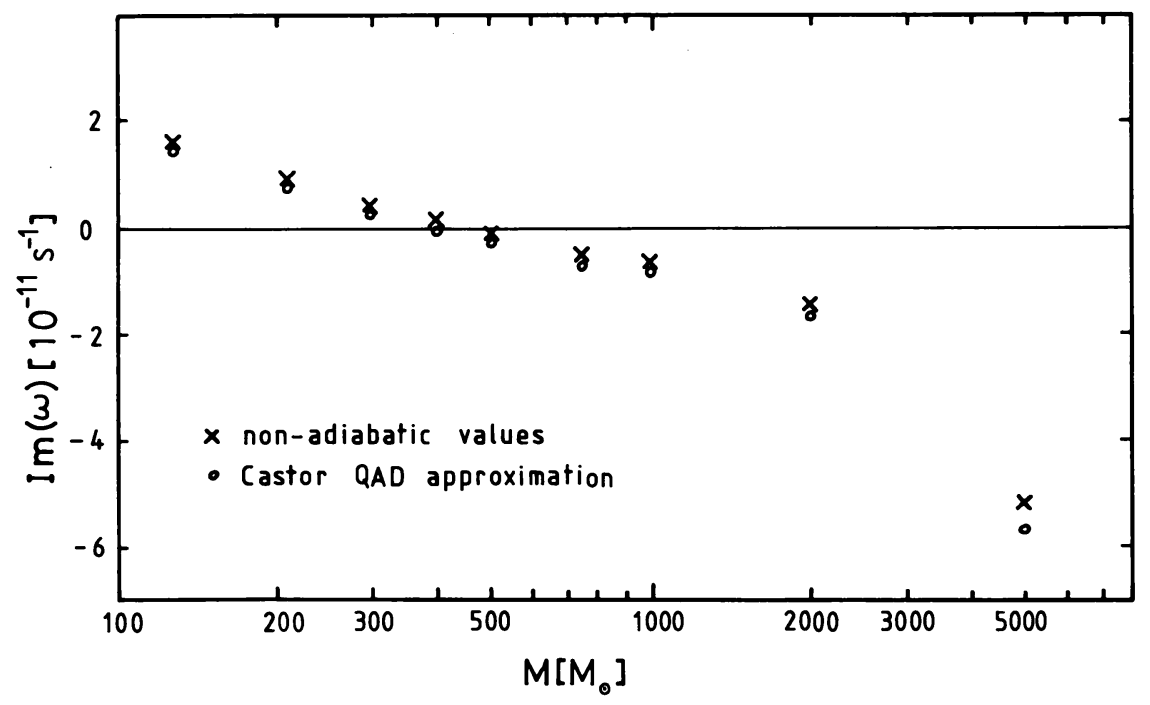

Fig. 1: The imaginary part of the eigenfrequency $\omega$ for the fundamental mode vs. the total stellar mass as obtained from the nonadiabatic calculation (crosses) and the quasiadiabatic CQAD approximation (circles).

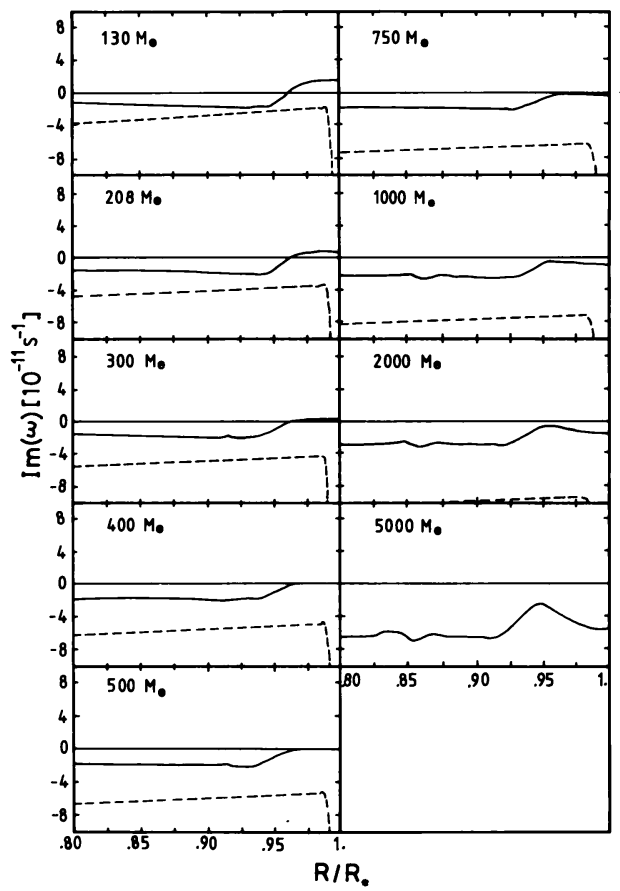

Fig. 2: The imaginary parts of the fundamental frequency from the CQAD (solid lines) and the LQAD (dashed lines) approximations as a function of the upper bound of the integrals over fractional radius in eqs. (1) and (2), respectively. 
For the CQAD approximation we observe a damping effect of the outermost layers for masses below the critical mass $\left(\sim 440 M_{\odot}\right)$ that changes the sign of $\operatorname{Im}(\omega)$. This damping occurs in the last $10 \%$ of the radius of the star. For the unstable cases we obtain two regions: one of negative damping (driving) just below the surface and a deeper region of positive damping. The effect of these regions almost cancel out completely in the high mass cases.

A detailed study of the effect of the outer nonadiabatic layers requires further investigation. However, it has become clear that the outer nonadiabatic layers are not only important but can actually determine the sign of $\operatorname{Im}(\omega)$.

We have thus found the important result that if we include the effects of the outer nonadiabatic layers the critical mass for stable hydrogen burning models increases to about $440 M_{\odot}$. For a complete description of the linear results see Klapp, Langer and Fricke (1986), where the significance of the results is discussed and extensions of this work to evolved stars and into the nonlinear regime are proposed.

Acknowledgement. The authors gratefully thank N. Baker, A.N. Cox, R. Kippenhahn, H.H. Loose, A.P. Odell, and A. Weiß for stimulating discussions. J. Klapp thanks the DAAD for a visiting fellowship and for hospitality at the Universitäts-Sternwarte Göttingen and at the Max Planck Institut für Physik und Astrophysik in Garching. He is also grateful to E. Nowotny for help in arranging the visit to Germany and to CONACYT for partial support under contract no. PCCBBAL-022508. N. Langer and K.J. Fricke are grateful to the Deutsche Forschungsgemeinschaft for partial support under grants Fr 325/15-2 and Fr 325/22-2.

\section{REFERENCES}

Castor, J.I.: 1971, Ap. J. 166, 109.

De Jager, C.: 1980, The Brightest Stars, Reidel, Dordrecht.

Humphreys, R.M.: 1982, in: Proc. ESO Workshop The Most Massive Stars (S. D'Odorico et al. eds.), Garching, p. 5.

Humphreys, R.M.: 1984, IAU-Symp. 105, 279.

Klapp, J., Langer, N., Fricke, K.J.: 1986, Ap. J., submitted.

Langer, N., El Eid, M.F., Fricke, K.J.: 1985, Astr. Ap. 145, 179.

Ledoux, P.: 1941, Ap. J. 94, 537.

Maeder, A.: 1982, in: Proc. ESO Workshop The Most Massive Stars (S. D'Odorico et al. eds.), Garching, p. 173.

Maeder, A.: 1985, Astr. Ap. 147, 300.

Massey, P., Hutchings, J.B.: 1983, Ap. J. 275, 578.

Noels, A., Masereel, C.: 1982, Astr. Ap. 105, 293.

Papaloizou, J.C.B.: 1973, M.N.R.A.S. 162, 143.

Schwarzschild, M., Härm, R.: 1959, Ap. J. 129, 637.

Stothers, R., Simon, N.R.: 1970, Ap. J. 160, 1019.

Ziebarth, K.: 1970, Ap. J. 162, 947. 